Review

\title{
Role of RHO family interacting cell polarization regulators (RIPORs) in health and disease: Recent advances and prospects
}

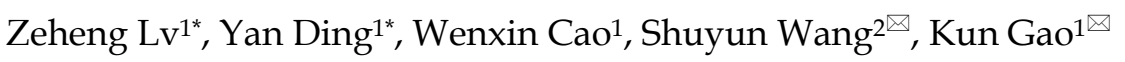 \\ 1. Department of Clinical Laboratory, Shanghai First Maternity and Infant Hospital, School of Medicine, Tongji University, Shanghai 200092, China. \\ 2. Department of Breast Surgery, Shanghai First Maternity and Infant Hospital, School of Medicine, Tongji University, Shanghai 200092, China. \\ *These authors contributed equally to this work. \\ $\triangle$ Corresponding authors: E-mail: kungao@tongji.edu.cn (K.G.); E-mail: 2000672@tongji.edu.cn (S.W.)
}

(C) The author(s). This is an open access article distributed under the terms of the Creative Commons Attribution License (https://creativecommons.org/licenses/by/4.0/). See http://ivyspring.com/terms for full terms and conditions.

Received: 2021.07.28; Accepted: 2021.11.27; Published: 2022.01.01

\begin{abstract}
The RHO GTPase family has been suggested to play critical roles in cell growth, migration, and polarization. Regulators and effectors of RHO GTPases have been extensively explored in recent years. However, little attention has been given to RHO family interacting cell polarization regulators (RIPORs), a recently discovered protein family of RHO regulators. RIPOR proteins, namely, RIPOR 1-3, bind directly to $\mathrm{RHO}$ proteins (A, B and C) via a RHO-binding motif and exert suppressive effects on RHO activity, thereby negatively influencing RHO-regulated cellular functions. In addition, RIPORs are phosphorylated by upstream protein kinases under chemokine stimulation, and this phosphorylation affects not only their subcellular localization but also their interaction with $\mathrm{RHO}$ proteins, altering the activation of $\mathrm{RHO}$ downstream targets and ultimately impacting cell polarity and migration. In this review, we provide an overview of recent studies on the function of RIPOR proteins in regulating RHO-dependent directional movement in immune responses and other pathophysiological functions.
\end{abstract}

Key words: RIPOR1; RIPOR2; RIPOR3; RHO GTPase; RHOA; RHOC; 14-3-3 protein; polarization; migration

\section{Introduction}

Cell migration plays a fundamental role in both normal physiological processes such as immune responses and abnormal pathologies such as tumor metastasis [1]. Establishing and maintaining frontrear polarity is integral to the movement of cells in specific directions. Cell polarization necessitates dynamic reorganization of the actin cytoskeleton, temporal changes in gene transcription, spatial localization of specific proteins, and reorientation of secretory trafficking [2].

The mechanisms underlying cell polarization and migration involve cooperation among multiple proteins, such as the small GTP-binding proteins RHO GTPases [3]. In mammals, 20 members of the RHO GTPase family have been identified and classified into eight subfamilies: RHO, RHOBTB, RHODF, RND, RHOUV, RHOH, CDC42 and RAC $[3,4]$. A better understanding of RHO GTPase function and how their activities are regulated is needed to elucidate the mechanisms of cell polarization and migration.

RHO GTPases act as molecular switches to control multiple cellular events by switching between an inactive GDP-bound state and an active GTPbound state [5-7]. This cycling activity is strictly regulated by three groups of factors: guanine nucleotide exchange factors (GEFs), which promote GDP/GTP exchange; GTPase-activating proteins (GAPs), which facilitate the hydrolysis of GTP to GDP; and guanine nucleotide dissociation inhibitors (GDIs), which prevent nucleotide exchange and regulate relocalization of RHO GTPases, thereby inhibiting their access to downstream effectors.

One of the most well-characterized subfamilies of RHO GTPases is the RHO subfamily, which contains three highly homologous proteins: RHOA, 
RHOB, and RHOC. In addition to other classic RHO regulators, recent studies have linked RHO family interacting cell polarization regulators (RIPORs) as novel partners of RHO subfamily members [8-11]. The RIPOR family is composed of RIPOR1 (also called FAM65A), RIPOR2 (also called FAM65B, C6orf32, KIAA0386, or PL48), and RIPOR3 (also called FAM65C).

In this review, we summarize recent research on the functions of RIPOR1 and RIPOR2 in the RHO signaling pathway, with a focus on advances in deciphering the mechanisms of RIPOR proteins in RHO-dependent directional movement in immune responses, tumor cell metastasis and other pathophysiological functions.

\section{The RIPOR family, a novel family of RHO-binding proteins}

\section{History of discovery}

In 1997, RIPOR2 was first cloned from differentiating cytotrophoblasts and reported as a novel gene with markedly elevated mRNA expression during the differentiation of DMSO-induced HL-60 cells [12]. RIPOR2 was then found to induce the formation of neurite-like protrusions in the murine myoblast cell line $\mathrm{C} 2 \mathrm{C} 12$ in 2007 and in quail myoblast QM-RSV cells in 2008 [13,14]; however, little is known about how RIPOR2 modulates cell morphogenesis. Later studies by Rougerie et al. and our research group demonstrated that RIPOR2 directly bound to RHOA in neutrophils [10]. RIPOR2 led to a reduction in active RHOA and RHOAmediated transcriptional activation, suggesting that RIPOR2 is a RHOA inhibitor. Moreover, inhibition of RHOA activity, as well as overexpression of RIPOR2, suppressed $\mathrm{T}$ cell migration, further confirming the involvement of RIPOR2-RHOA in cell polarization [8]. Collectively, these findings demonstrated that RIPOR2 is involved in regulating $T$ cell and neutrophil migration through RHOA signaling. Since these findings, an increasing number of studies have been carried out to decipher the important roles of RIPORs in cell migration and polarization. In 2016, Mardakheh et al. revealed that RIPOR1 directly interacted with active RHOA, RHOB and RHOC [11]. In contrast, the functions of RIPOR3 have not yet been elucidated.

\section{Structural basis for the regulation of the RHO subfamily by RIPOR proteins}

RIPOR1, RIPOR2 and RIPOR3 share an identical RHO-binding motif in the N-terminal region (Figure 1), which shows moderate similarity to the RHOA-binding homology regions (HRs) in several known RHOA effectors, such as protein kinase $\mathrm{N}-1$, -2 , and -3 (PKN1, PKN2, PKN3), Rhotekin (RTKN1), and Rhophilin (RHPN1). The invariant glycine (Gly155 in RIPOR2) and alanine (Ala156 in RIPOR2) are highly conserved among these RHOA effectors. Except for these two amino acids, the amino acids within the RHO-binding motif (RBM, aa 138-210) in RIPORs are also highly homologous, especially Arg151 and Leu152. Mutation of Arg151 and Leu152 to Ala or of Gly155 and Ala156 to Arg in RIPOR2 abolish the interaction between RIPOR2 and RHOA [10]. The N-terminal parts of RIPORs are evolutionarily conserved, and more than $70 \%$ identity is observed among the RIPORs. In addition, a RIPOR1 mutant lacking the $\mathrm{N}$-terminus failed to interact with RHOA and was unable to inhibit RHOA activity [10]. These observations highlighted the importance of the N-terminal segment of RIPOR proteins. RIPOR3 shows high homology with RIPOR1/2 in the RBM, implying that RIPOR3 is a potential interacting protein of RHOA. Indeed, we confirmed this interaction between RIPOR3-RHO proteins through the RBM by coimmunoprecipitation assays (data not published). Finally, a brief phylogenetic analysis of RIPORs suggested that RIPORs are encoded only in vertebrate genomes and the RBM is highly conserved in all RIPORs irrespective of species (data not shown).

Analysis of the protein sequences of RIPORs also revealed some unique structures among RIPORs. For example, RIPOR1 contains a HEAT repeat domain in its C-terminal region, although the function of this domain has not been elucidated (Figure 1).

\section{Posttranslational modifications (PTMs) of RIPOR2}

Based on PhosphoSitePlus data, the three RIPORs are potentially phosphorylated. Upon chemoattractant stimulation, RIPOR2 showed a motility shift and was stabilized in neutrophils. This phenomenon was phenocopied in cells treated with the phosphatase inhibitor okadaic acid [10]. However, deletion of phospholipase C (PLC) $\beta 2 / \beta 3$ and phosphatidylinositol-3-kinase $(\mathrm{PI} 3 \mathrm{~K}) \gamma$ impaired the motility shift of RIPOR2 [10]. Therefore, chemokines induce RIPOR2 phosphorylation in part through PKC and AKT [9-10,15]. Treatment with the proteasome inhibitor MG132 stabilized RIPOR2, implying that its turnover is controlled by the proteasomal pathway [9-10]. These findings shed light on the underlying mechanisms by which RIPOR2 phosphorylation might impede its degradation by the proteasome. Moreover, in cardiomyocytes, RIPOR2 can be phosphorylated by phosphatase and tensin homologinduced putative kinase 1 (PINK1) to participate in autophagy [15]. 




Figure 1. The domain architecture of RIPOR family and sequence alignment of amino acids of the RBM in RIPORs (RIPOR1: NP_078795.2,RIPOR2: NP_055537.2,RIPOR3: NP_543019.2) with PKN1 (NP_998725.1), PKN2 (NP_006247.1), PKN3 (NP_037487.2) and the HR of RTKN (NP_149035.1) and RHPN1 (NP_443156.2).

Notably, stimulation with the chemokine CCL19 was found to disrupt the RIPOR2-RHOA interaction, an effect that was abrogated by phosphatase inhibitor treatment [9], implying that chemokine-induced RIPOR2 phosphorylation decreases the interaction between RIPOR2 and RHOA.

RIPOR2 phosphorylation also alters its subcellular localization during cell migration. Stimulation with the chemokine fMLP induced the accumulation of RIPOR2 at the leading edge of neutrophils. However, the distribution of the nonphosphorylated RIPOR2 S5A mutant (incapable of phosphorylation by PKC/AKT) was not affected by chemokine stimulation [10]. Interestingly, phosphorylation was found to result in relocalization of RIPOR2 from the plasma membrane to the cytosol, thereby disrupting the interaction of RIPOR2 with RHOA [9]. In summary, phosphorylation of RIPOR2 not only disrupts the RIPOR2-RHOA interaction but also impacts the subcellular localization of RIPOR2.

Data from the PhosphoSitePlus database indicate that the RIPOR1 and RIPOR3 proteins contain several phosphorylation sites [16]. For this reason, we speculate that both RIPOR1 and RIPOR3 may be phosphorylated, although additional experiments are warranted.

Other PTMs, including ubiquitination of RIPOR1/2, acetylation of RIPOR2/3 and arginine methylation of RIPOR3, have also been deduced from high-throughput mass spectrometry data [16]. However, the precise cellular consequence of these PTMs on RIPORs remains elusive.

\section{4-3-3 family members are RIPOR-binding partners}

14-3-3 family proteins (also called tyrosine 3monooxygenase/tryptophan 5-monooxygenase activation proteins (YWHAs)) are highly conserved proteins that are widely expressed in eukaryotic cells $[17,18]$. 14-3-3 family members were recently identified as novel interacting proteins of RIPOR1/2 $[10,11]$. These proteins bind to Ser/Thrphosphorylated proteins as hetero- or homodimers by recognizing three types of conserved motifs: RSXpSXP, RXY/FXpSXP, and pSX1-2-COOH [17,19]. Protein sequence analysis showed that RIPORs have many potential 14-3-3 binding sites (Figure 2). The 14-3-3 consensus sequences in RIPORs also match the substrate motifs for many protein kinases. Our work showed that RIPOR2 can be phosphorylated by PKC and AKT at serine residues 21, 37, 341, 523, and 535 in regions that match the 14-3-3 consensus sequences. Not surprisingly, the RIPOR2 S5A mutant showed a much lower affinity for 14-3-3 proteins [10]. As regulatory molecules, 14-3-3 proteins modulate the conformation, activity, and subcellular localization of their interacting partners [20]. Indeed, overexpression of 14-3-3 proteins led to higher levels of RIPOR2, implying that 14-3-3 proteins promote RIPOR2 stabilization [10]. In addition, several 14-3-3 proteins interacted with RIPOR1 in a RHO-dependent manner [11], although the effects of 14-3-3 proteins on RIPOR1 have not been examined. 




Figure 2. The potential 14-3-3 binding motifs in RIPORs.

\section{The pathophysiological functions of RIPORs}

\section{RIPOR2 and immune cell function}

The biological significance of the role of RHO in $\mathrm{T}$ cell development, activation, and migration is well documented [21-23]. RIPOR2 functions in the adhesion, polarization, and migration of $\mathrm{T}$ cells in a RHOA-dependent manner. Depletion of RIPOR2 by small interfering RNAs increased the percentage of cells exhibiting arrest in vitro. A similar effect was further verified in lymphocyte arrest on blood vessel endothelial cells [8]. Inhibition of RHOA activity, as well as overexpression of RIPOR2, was found to impede T cell migration. In contrast, the RIPOR $2 \Delta 113$ mutant, which was unable to bind RHOA, did not exert this effect [8]. RIPOR2 was found to inhibit GTP loading on RHOA and preferentially bind active RHOA proteins to block RHOA activity in neutrophils [10]. These observations suggest that the function of RIPOR2 in cell migration is mediated mainly through RHOA inhibition.

In addition to its role in T cell migration, RIPOR2 is reported to act as a negative regulator of cell proliferation in a RHOA-independent manner. The RIPOR2 S5A mutant but not RIPOR2 RL151-152AA lost its antiproliferative effect [25]. Previous studies showed that 14-3-3s are binding partners of HDAC6, a cytoplasmic deacetylase that promotes cancer cell proliferation in a deacetylase activity-dependent manner. HDAC6 regulated 14-3-3 interactions in a deacetylation activity-dependent manner [26]. Furthermore, RIPOR2 inhibited the deacetylase activity of HDAC6. Consequently, phosphorylated RIPOR2 interacted with 14-3-3 and HDAC6 to form a tripartite complex [24-25], and this complex suppressed HDAC6 activity, resulting in disruption of the mitotic spindle formation and mitotic failure in resting T cells [25].

The active forkhead box O1 (FOXO1) transcription factor is required for maintaining the quiescence of $\mathrm{T}$ cells by promoting RIPOR2 transcription and expression $[8,25]$. In $\mathrm{T}$ cells, $\mathrm{T}$ cell receptor (TCR) stimulation led to cytoplasmic localization of FOXO1 in a PI3K-AKT pathwaydependent manner [27]. Notably, under TCR stimulation, RIPOR2 expression was reduced upon TCR activation [25]. Thus, an increased cytoplasmic localization of FOXO1 upon TCR stimulation can be hypothesized to negatively regulate the expression of RIPOR2, which is essential for T cell proliferation.

In summary, these results indicate that RIPOR2 plays roles in the migration and proliferation of $\mathrm{T}$ cells by modulating the functions of RHOA and HDAC6, respectively.

\section{RIPOR2 and autophagy}

Autophagy is a highly conserved process in all eukaryotes in which intracellular components are transported to lysosomes for degradation and recycling $[28,29]$. Distinct from microautophagy and 
chaperone-mediated autophagy, macroautophagy is well established and involves the formation of autophagosomes [29]. Autophagosomes carry and deliver cargo to lysosomes and then fuse with lysosomes to form autolysosomes, in which the contents are subsequently degraded [28-29]. Autophagy functions as a major cytoprotective process by maintaining cellular homeostasis and recycling cytoplasmic contents, and dysfunction of autophagy has been associated with multiple human diseases [30-31].

Zhou et al. reported that RIPOR2 is involved in autophagy. A circRNA termed autophagy-related circular RNA (ACR) activates PINK1 expression by directly binding to DNMT3B and blocking DNMT3B-mediated promoter methylation of PINK1. RIPOR2 is a downstream target of PINK1. PINK1 phosphorylates RIPOR2 at serine 46, and phosphorylated RIPOR2 inhibits autophagy and cell death in cardiomyocytes [15]. However, it remains unclear whether phosphorylated RIPOR2 governs autophagy in a RHOA-dependent manner.

\section{RIPOR2 and muscle cell differentiation}

During the development of skeletal muscle, myoblasts from myotubes recognize other myoblasts, adhere, and fuse to form multinucleated myotubes; this process is critical in cellular differentiation and fusion [14,32]. Multiple proteins, such as myogenic transcription factor (MRF4), neural cell adhesion molecule (N-CAM), and M-cadherin, are involved in these tightly regulated processes [13,33-34]. Accumulating evidence suggests that RIPOR2 also functions as one of the fundamental regulators of myogenic differentiation $[14,35]$.

In 2007, RIPOR2 was first characterized in human fetal muscle cell differentiation [14]. RIPOR2 silencing reduced the protein level of myogenin, a marker of early myogenic differentiation, and abrogated primary human fetal muscle cell differentiation and fusion [14]. RIPOR2 was also implicated in the formation of filopodia, which has been reported to be critical in key morphogenesis events at certain stages of muscle development $[14,36]$. RIPOR2 binds to HDAC6 and dysferlin, the protein mutated in limb girdle muscular dystrophy 2B. The RIPOR2-HDAC6-dysferlin trimeric complex is transient, and RIPOR2 expression is necessary for the complex to form. Treatment of myogenic cells with pan-HDAC or HDAC6-specific inhibitors alters RIPOR2 expression. Inhibition of RIPOR2 expression in developing zebrafish results in abnormal muscle with low birefringence and myoseptal tears and in increased embryonic lethality [35].

RHOA has also been implicated in myoblast fusion [32]. Active RHOA was found to induce lysosomal degradation of M-cadherin, one of the cell adhesion molecules involved in the fusion of myoblasts [33,37], and abrogated the interaction between M-cadherin and its partner p120-catenin, leading to inhibition of myoblast fusion [32]. Given that RIPOR2 expression increases during muscle cell differentiation and that the amount of active RHOA decreases during myoblast fusion, it is possible that the effects of RIPOR2 on myoblast fusion events in membrane protuberances depend on RHOA inhibition. Therefore, RIPOR2 is likely to act as an inducible repressor of RHOA activity to promote cell-cell fusion during muscle or placenta formation.

N-CAM, another RIPOR2 binding partner, is also related to myoblast fusion [13,37-38]. However, the functional impact of the N-CAM-RIPOR2 interaction on myoblast fusion is still unknown.

\section{RIPOR2 and hair cell functions}

Cochlear hair cells are crucial for hearing due to their role in converting sound-induced vibration into electrical signals. This function relies on the specialized bundle of stereocilia at the apical surface of the hair cell [39]. Stereocilia are tapered at their base near their site of insertion into the apical hair cell and are formed into a unique stairstep shape [40]. Accumulating evidence suggests that the structure and dynamic properties of the actin core are essential for the functions of stereocilia [40].

Several studies have indicated that a loss-offunction mutation in RIPOR2 is correlated with hearing [41-44]. Diaz-Horta et al. identified a splice site mutation (c.102-1G>A) in the RIPOR2 gene (MIM611410) that completely cosegregated with the phenotype in a large consanguineous Turkish family with recessive nonsyndromic, prelingual, profound hearing loss [41]. The enrichment of RIPOR2 in the stereocilia of both inner and outer hair cells may suggest that a lack of functional RIPOR2 can hinder the development of the mechanotransduction apparatus. Moreover, knockdown of RIPOR2 causes hearing loss in zebrafish. A later report showed that RIPOR2 was localized in the basal taper of the mechanically sensitive stereocilia of hair cells, where oligomeric RIPOR2 interacted with RHOC to form the circumferential ring, an essential component of the taper region [42]. RIPOR2 negatively regulates the structure and orientation of the hair cell stereociliary bundle via a mechanism based partially on the RIPOR2-nonmuscle myosin heavy chain 9 (MYH9) interaction to stabilize MYH9 [43]. MYH9-mediated coupling between microtubules and actomyosin is involved in the formation of nonmuscle myosin II (NMII) [45]. Given that RIPOR2 inhibits HDAC6 
activity and that HDCA6 destabilizes microtubule structures by promoting a-tubulin deacetylation, it might be possible that RIPOR2 contributes to microtubule flexibility rather than lengthening kinocilia in inner and outer hair cells $[43,46]$.

However, it might be possible that RIPOR2 functions in maintaining normal hearing through mechanisms other than affecting the MYH9 abundance or inhibiting HDAC6 activity [44]. An inframe deletion (c.1696_1707del) mutation in RIPOR2 has been linked with hearing loss [44]. Strikingly, this mutation did not alter RIPOR2's function in filopodia formation, suggesting that a RHO-independent mechanism may exist. Further studies are needed to clarify the RHO-dependent and RHO-independent mechanisms of RIPOR2 in mediating hair cell function.

\section{RIPOR2 and cancer}

According to data from The Cancer Genome Atlas [47], the expression of RIPOR2 is downregulated compared to that in normal tissues in the majority of cancer types, especially in uterine corpus endometrial carcinoma and diffuse large B-cell lymphoma. However, the precise roles of RIPOR2 in tumorigenesis and progression are still poorly understood.

Zhang $\mathrm{K}$ et al. reported that RIPOR2 was upregulated in PC3 holoclones with cancer stem cell characteristics. PC3 cells, a line of androgenindependent human prostate cancer cells, were divided into three morphologically distinct colonies: holoclones, meroclones, and paraclones. Compared with meroclones and paraclones, holoclones exhibited increased self-renewal activity and proliferation capability. In addition, gene expression patterns differed among the three colony types. The expression of RIPOR2 was significantly upregulated in PC3 holoclones. Furthermore, PC3 prostate tumorinitiating cells with the molecular profile RIPOR2high / melanotransferrin (MFI2) ${ }^{\text {low }} /$ LEF1 ${ }^{\text {low }}$ increased tumor angiogenesis [48]. Despite these findings, the molecular mechanisms underlying RIPOR2-related angiogenesis are still unknown.

\section{The pathophysiological functions of RIPOR 1 and RIPOR3}

Among the RIPOR family members, RIPOR2 is the best studied. As described above, RIPOR2 is involved in a plethora of physiological and pathological processes, such as immune cell polarization, migration, and autophagy (Figure 3). Despite their structural similarities, RIPOR1 and RIPOR3 show potentially distinct functional features relative to RIPOR2.

To better understand the potential functions of the RIPOR proteins, we examined the mRNA expression patterns of RIPORs in 79 human tissues [49] (Figure 4). RIPORs are differentially expressed in human tissues. RIPOR2 is predominantly expressed in immune cells, including T cells, B cells, NK cells, monocytes and myeloid cells. Our previous study showed that the RIPOR2 mRNA level is much higher in bone marrow neutrophils than in other cell types [10]. In contrast, RIPOR1 and RIPOR3 are ubiquitously expressed in the majority of tissues. The distinct expression patterns of RIPORs suggest the different biological functions performed by these proteins. RIPOR1 regulates Golgi apparatus reorientation during HeLa cell migration. It functions as a scaffold protein that links $\mathrm{RHO}$ proteins to Golgi-localizing proteins (Cerebral Cavernous Malformation-3 (CCM3) and mammalian STE20-like protein kinase 3 (MST3), and MST4), resulting in relocalization of Golgi-localized proteins from the Golgi apparatus toward the leading edge of cells during directional cell migration. In response to wounding, the Golgi apparatus was found to be reoriented in HeLa cells at the edge of the wound. Depletion of RIPOR1 impeded Golgi apparatus reorientation and directional migration of HeLa cells following wounding. This effect was consistent with that of RHOA activity inhibition [11]. Therefore, the divergent interacting proteins of RIPORs may account for their distinct functions (Table 1).

Table 1. Interactors of RIPOR Family

\begin{tabular}{|c|c|c|c|c|}
\hline Protein & Interactors & Process/Pathway Affected & Cells & Reference \\
\hline RIPOR1 & RHOA & $\begin{array}{l}\text { linking active RHO subfamily } \\
\text { and Golgi-localizing proteins to } \\
\text { regulate Golgi reorientation }\end{array}$ & HeLa cells & [11] \\
\hline \multirow[t]{7}{*}{ RIPOR2 } & RHOA & $\begin{array}{l}\text { Inhibiting RHOA activity to } \\
\text { negatively regulate immune cell } \\
\text { migration }\end{array}$ & $\begin{array}{l}\text { T lymphocytes } \\
\text { Primary } \\
\text { neutrophils }\end{array}$ & {$[8-10]$} \\
\hline & $\begin{array}{l}\text { HDAC6, } \\
14-3-3\end{array}$ & $\begin{array}{l}\text { Interacting with HDAC6 } \\
\text { deacetylase and } 14-3-3 \text { to inhibit } \\
\text { T-cells proliferation }\end{array}$ & T lymphocytes & [25] \\
\hline & $\begin{array}{l}\text { HDAC6, } \\
\text { Dysferlin }\end{array}$ & $\begin{array}{l}\text { Interacting with HDAC6 and } \\
\text { dysferlin to promote myogenic } \\
\text { differentiation }\end{array}$ & $\begin{array}{l}\text { Primary } \\
\text { muscle cells }\end{array}$ & [35] \\
\hline & HDAC6 & $\begin{array}{l}\text { Inhibiting HDAC6 activity to } \\
\text { maintain the structure and } \\
\text { function of the auditory hair cell } \\
\text { bundle }\end{array}$ & Hair cells & [43] \\
\hline & MYH9 & $\begin{array}{l}\text { Stabilizing non-muscular } \\
\text { myosin heavy chain } 9(\mathrm{MYH} 9) \text { to } \\
\text { maintain the structure and } \\
\text { function of the auditory hair cell } \\
\text { bundle }\end{array}$ & Hair cells & [43] \\
\hline & RHOC & $\begin{array}{l}\text { Interacting with RHOC to form } \\
\text { a RIPOR2 ring-like structure } \\
\text { critical for mechanosensory hair } \\
\text { cell function }\end{array}$ & Hair cells & [42] \\
\hline & $\mathrm{N}-\mathrm{CAM}$ & Interacting with NCAM & - & [13] \\
\hline RIPOR3 & unknown & - & - & - \\
\hline
\end{tabular}




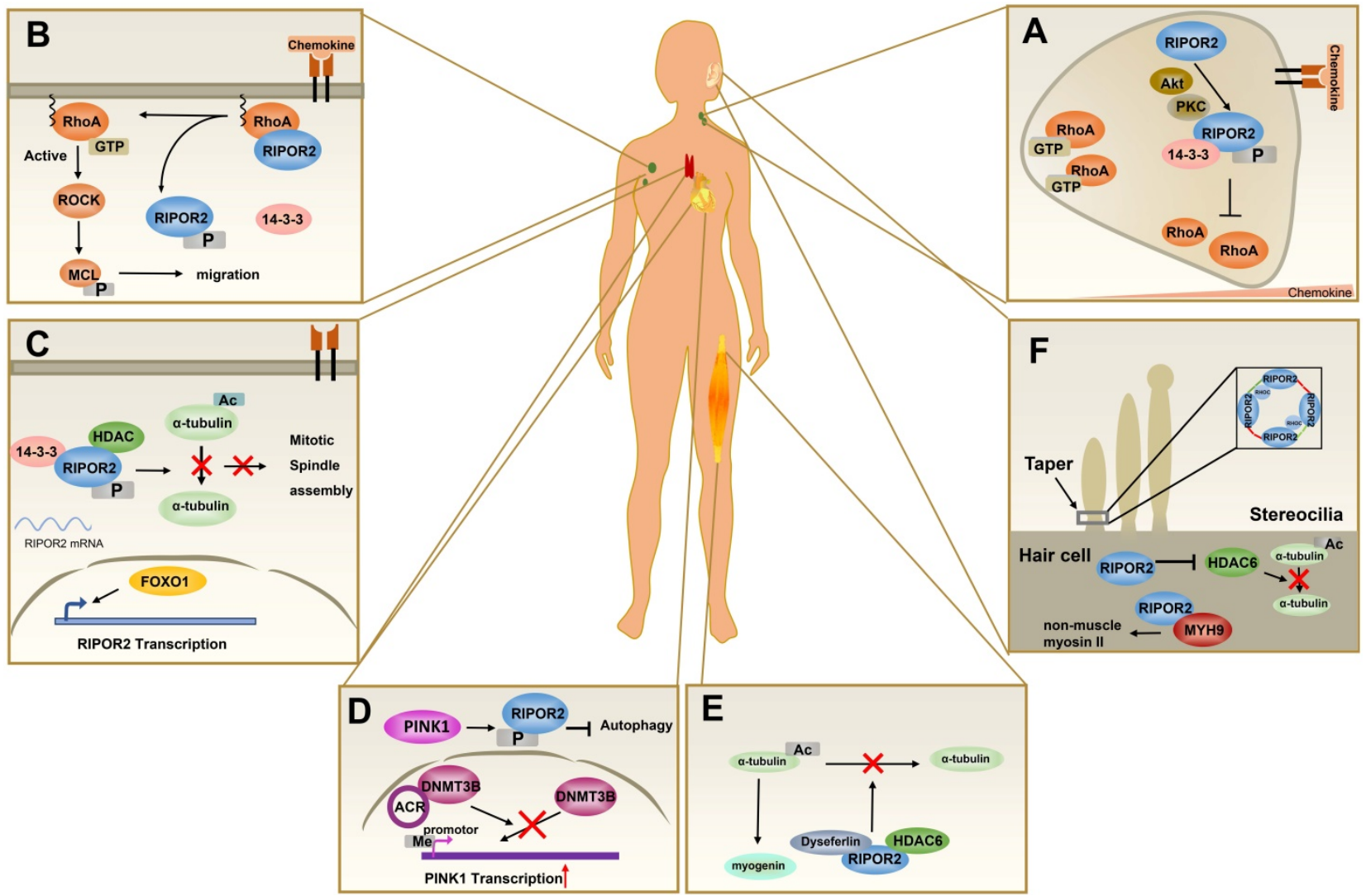

Figure 3. The pathophysiological functions of RIPOR2. (A) In primary neutrophils, the front localization of phosphorylated RIPOR2 stabilized by 14-3-3 leads to unequal distribution of active RHOA. (B) In resting T cells, RIPOR2 combines with RHOA and exerts suppressive effects on its activity at the plasma membrane to maintain immune cells in a quiescent state. Upon chemokine stimulation, 14-3-3 interacts with phosphorylated RIPOR2 by protein kinases, breaking the RIPOR2-RHOA interaction and relieving RHOA inhibition. (C) In resting T cells, FOXO1 upregulates RIPOR2 protein level, and then phosphorylated RIPOR2 binds to 14-3-3 and HDAC6 to form tricomplex, inhibiting deacetylation of $\alpha$-tubulin and thereby discouraging mitotic spindle assembly. (D) By interacting with DNMT3B, ACR suppresses DNA methylation of PINK1 to upregulate the expression of PINK1, increasing RIPOR2 phosphorylation, which contributes to autophagy inhibition and cell death in cardiomyocytes. (E) Tricomplex formed by HDAC6, RIPOR2, and dysferlin inhibits the deacetylation activity of HDAC6, resulting in the accumulation of acetylated $\alpha$-tubulin, which is believed to promote muscle cell differentiation. (F) RIPOR2 is located in the basal taper of mechanically sensitive stereocilia of hair cells, where it interacts with RhoC to form a circumferential ring--RIPOR2 oligomerization, a structure necessary for the taper region. As well as stabilizing MYH9 in hair cells, RIPOR2 inhibits HDAC6 activity in order to regulate stereociliary bundle structure.

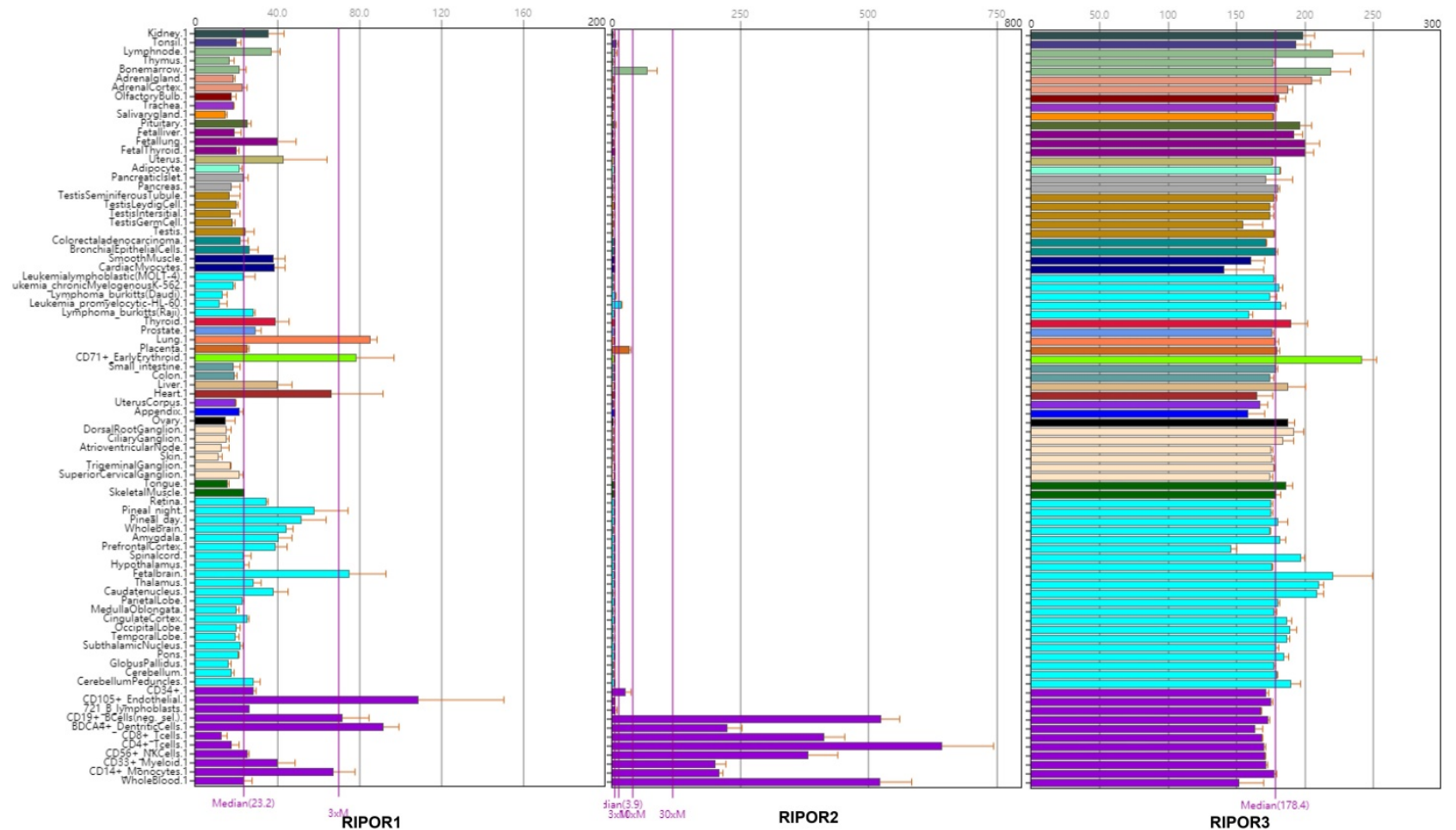

Figure 4. High-density oligonucleotide arrays are used to examine patterns of gene expression in a panel of 79 human tissues. The data is from BioGPS. 
Analysis of RNA-seq data in The Cancer Genome Atlas showed that RIPOR1 is upregulated in gastrointestinal tumors such as cholangiocarcinoma, colon adenocarcinoma, esophageal carcinoma, and liver hepatocellular carcinoma. Intriguingly, the expression level of RHOA in these gastrointestinal tumors is also increased [47]. Given that RHOA is generally considered an oncogene [50], we hypothesize that RIPOR1 may act as an oncogene or tumor suppressor in a context-dependent manner, although further investigations are warranted in the future.

Likewise, little is known about the biological functions of RIPOR3 in cells. As no studies have yet examined RIPOR3, we can only speculate from its mRNA expression profile that the functions of RIPOR3 may differ from those of RIPOR1 and RIPOR2. Further investigations are needed to decipher the biological functions of RIPOR3 and determine whether these functions are altered in pathological conditions.

\section{Conclusions}

These findings illustrate the unity and versatility of the RIPOR family in health and disease. As a first insight, the RIPORs share many similarities in amino acid sequences, protein interaction partners, and biological functions. Moreover, the differences in their roughly homologous amino acid sequences might account for their varying functions through interactions with proteins in addition to RHO (A, B, and $\mathrm{C}$ ).

Here, we highlight the roles of RIPORs in physiological and pathological contexts - especially roles linked with the actin cytoskeleton, including cell polarization, migration, adhesion, and organelle trafficking - based on studies conducted in this decade. Currently, it is clear that the regulation of RHO GTPase functions is shared by three members, but their other molecular functions are still poorly understood. Given their sequence divergence and tissue-specific expression, RIPORs are expected to function in diverse biological processes. More research is needed to clearly clarify the roles of RIPORs in homeostasis and disease.

\section{Abbreviations}

RIPOR: the RHO family interacting cell polarization regulators; GEFs: guanine nucleotide exchange factors; GAPs: GTPase activating proteins; GDIs: guanine nucleotide dissociation inhibitors; MST4: mammalian STE20-like protein kinases 4; PKN1: protein kinase N-1; RTKN1: Rhotekin; RHPN1: Rhophilin; PLC: Phospholipase C; PI3K: phosphatidyl-inositol-3-kinase; PINK1: phosphatase and tensin homolog-induced putative kinase 1 ; HDAC6: histone deacetylase 6; FOXO1: forkhead box O1; TCR: $\mathrm{T}$ cell receptor; DNMT3B: DNA methyltransferase 3 beta; MRF4: myogenic transcription factor; N-CAM: neural cell adhesion molecule; MYH9: non-muscular myosin heavy chain 9; NMII: non-muscle myosin II; MFI2: melanotransferrin; CCM3: Cerebral Cav-ernous Malformation-3 protein; MST3: Mammalian STE20-like protein kinases 3.

\section{Acknowledgements}

\section{Author Contributions}

All authors contributed to manuscript conceptualization and preparation. All authors have read and agreed to the published version of the manuscript.

\section{Funding}

This work was in part supported by the National Natural Science Foundation of China (No. 91954106, 81872109 to K.G.), the Natural Science Foundation of Shanghai (No. 18ZR1430100 to K.G.) and the Open Research Fund of State Key Laboratory of Genetic Engineering, Fudan University (No. SKLGE-2111).

\section{Availability of data and materials}

The datasets generated and analyzed during the current study are available from the corresponding authors on reasonable request.

\section{Competing Interests}

The authors have declared that no competing interest exists.

\section{References}

1. Trepat X, Chen Z, Jacobson K. Cell migration. Compr Physiol. 2012; 2: 2369-92.

2. Inagaki N, Katsuno $\mathrm{H}$. Actin Waves: Origin of Cell Polarization and Migration? Trends Cell Biol. 2017; 27: 515-26.

3. Ridley AJ. Rho GTPase signalling in cell migration. Curr Opin Cell Biol. 2015; 36: 103-12.

4. Narumiya S, Thumkeo D. Rho signaling research: history, current status and future directions. FEBS Lett. 2018; 592: 1763-76.

5. Clayton NS, Ridley AJ. Targeting Rho GTPase Signaling Networks in Cancer. Front Cell Dev Biol. 2020; 8: 222.

6. Hall A. Rho family GTPases. Biochem Soc Trans. 2012; 40: 1378-82.

7. Iden S, Collard JG. Crosstalk between small GTPases and polarity proteins in cell polarization. Nat Rev Mol Cell Biol. 2008; 9: 846-59.

8. Rougerie P, Largeteau Q, Megrelis L, Carrette F, Lejeune T, Toffali L, et al. Fam65b is a new transcriptional target of FOXO1 that regulates RHOA signaling for T lymphocyte migration. J Immunol. 2013; 190: 748-55.

9. Megrelis L, El Ghoul E, Moalli F, Versapuech M, Cassim S, Ruef N, et al. Fam65b Phosphorylation Relieves Tonic RHOA Inhibition During T Cell Migration. Frontiers in Immunology. 2018; 9.

10. Gao K, Tang W, Li Y, Zhang P, Wang D, Yu L, et al. Front-signal-dependent accumulation of the RHOA inhibitor FAM65B at leading edges polarizes neutrophils. J Cell Sci. 2015; 128: 992-1000.

11. Mardakheh FK, Self A, Marshall CJ. RHO binding to FAM65A regulates Golgi reorientation during cell migration. J Cell Sci. 2016; 129: 4466-79.

12. Dakour J, Li H, Morrish DW. PL48: a novel gene associated with cytotrophoblast and lineage-specific HL-60 cell differentiation. Gene. 1997; 185: 153-7. 
13. Hirayama E, Kim J. Identification and characterization of a novel neural cell adhesion molecule (NCAM)-associated protein from quail myoblasts: relationship to myotube formation and induction of neurite-like protrusions. Differentiation. 2008; 76: 253-66.

14. Yoon S, Molloy MJ, Wu MP, Cowan DB, Gussoni E. C6ORF32 is upregulated during muscle cell differentiation and induces the formation of cellular filopodia. Dev Biol. 2007; 301: 70-81.

15. Zhou LY, Zhai M, Huang Y, Xu S, An T, Wang YH, et al. The circular RNA ACR attenuates myocardial ischemia/reperfusion injury by suppressing autophagy via modulation of the Pink1/ FAM65B pathway. Cell Death Differ. 2019; 26: 1299-315.

16. Hornbeck PV, Zhang B, Murray B, Kornhauser JM, Latham V, Skrzypek E. PhosphoSitePlus, 2014: mutations, PTMs and recalibrations. Nucleic Acids Res. 2015; 43: D512-20.

17. Fu H, Subramanian RR, Masters SC. 14-3-3 proteins: structure, function, and regulation. Annu Rev Pharmacol Toxicol. 2000; 40: 617-47.

18. Mhawech P. 14-3-3 proteins--an update. Cell Res. 2005; 15: 228-36.

19. Yaffe MB, Rittinger K, Volinia S, Caron PR, Aitken A, Leffers $H$, et al. The structural basis for 14-3-3:phosphopeptide binding specificity. Cell. 1997; 91: 961-71.

20. Gardino AK, Yaffe MB. 14-3-3 proteins as signaling integration points for cell cycle control and apoptosis. Semin Cell Dev Biol. 2011; 22: 688-95.

21. Pernis AB. Rho GTPase-mediated pathways in mature CD4+ T cells. Autoimmun Rev. 2009; 8: 199-203.

22. Saoudi A, Kassem S, Dejean A, Gaud G. Rho-GTPases as key regulators of T lymphocyte biology. Small GTPases. 2014; 5 .

23. Rougerie P, Delon J. Rho GTPases: masters of $\mathrm{T}$ lymphocyte migration and activation. Immunol Lett. 2012; 142: 1-13.

24. Wickstrom SA, Masoumi KC, Khochbin S, Fassler R, Massoumi R. CYLD negatively regulates cell-cycle progression by inactivating HDAC6 and increasing the levels of acetylated tubulin. EMBO J. 2010; 29: 131-44.

25. Froehlich J, Versapuech M, Megrelis L, Largeteau Q, Meunier S, Tanchot C, et al. FAM65B controls the proliferation of transformed and primary $\mathrm{T}$ cells. Oncotarget. 2016; 7: 63215-25.

26. Mortenson JB, Heppler LN, Banks CJ, Weerasekara VK, Whited MD, Piccolo SR, et al. Histone deacetylase 6 (HDAC6) promotes the pro-survival activity of 14-3-3zeta via deacetylation of lysines within the 14-3-3zeta binding pocket. J Biol Chem. 2015; 290: 12487-96.

27. Fabre S, Lang V, Harriague J, Jobart A, Unterman TG, Trautmann A, et al. Stable activation of phosphatidylinositol 3-kinase in the T cell immunological synapse stimulates Akt signaling to FoxO1 nuclear exclusion and cell growth control. J Immunol. 2005; 174: 4161-71.

28. Levy JMM, Towers CG, Thorburn A. Targeting autophagy in cancer. Nat Rev Cancer. 2017; 17: 528-42.

29. Parzych KR, Klionsky DJ. An overview of autophagy: morphology, mechanism, and regulation. Antioxid Redox Signal. 2014; 20: 460-73.

30. Levine B, Kroemer G. Biological Functions of Autophagy Genes: A Disease Perspective. Cell. 2019; 176: 11-42.

31. Racanelli AC, Kikkers SA, Choi AMK, Cloonan SM. Autophagy and inflammation in chronic respiratory disease. Autophagy. 2018; 14: 221-32.

32. Charrasse S, Comunale F, Grumbach Y, Poulat F, Blangy A, Gauthier-Rouviere C. RHOA GTPase regulates M-cadherin activity and myoblast fusion. Mol Biol Cell. 2006; 17: 749-59.

33. Kaufmann U, Martin B, Link D, Witt K, Zeitler R, Reinhard S, et al. M-cadherin and its sisters in development of striated muscle. Cell Tissue Res. 1999; 296: 191-8.

34. Krauss RS. Regulation of Skeletal Myoblast Differentiation by Drebrin. Adv Exp Med Biol. 2017; 1006: 361-73.

35. Balasubramanian A, Kawahara G, Gupta VA, Rozkalne A, Beauvais A, Kunkel LM, et al. Fam65b is important for formation of the HDAC6-dysferlin protein complex during myogenic cell differentiation. FASEB J. 2014; 28: 2955-69.

36. Segal D, Dhanyasi N, Schejter ED, Shilo BZ. Adhesion and Fusion of Muscle Cells Are Promoted by Filopodia. Dev Cell. 2016; 38: 291-304.

37. Ishido M, Uda M, Masuhara M, Kami K. Alterations of M-cadherin, neural cell adhesion molecule and beta-catenin expression in satellite cells during overload-induced skeletal muscle hypertrophy. Acta Physiol (Oxf). 2006; 187: 407-18.

38. Charlton CA, Mohler WA, Blau HM. Neural cell adhesion molecule (NCAM) and myoblast fusion. Dev Biol. 2000; 221: 112-9.

39. Goutman JD, Elgoyhen AB, Gomez-Casati ME. Cochlear hair cells: The sound-sensing machines. FEBS Lett. 2015; 589: 3354-61.

40. Manor U, Kachar B. Dynamic length regulation of sensory stereocilia. Semin Cell Dev Biol. 2008; 19: 502-10.

41. Diaz-Horta O, Subasioglu-Uzak A, Grati M, DeSmidt A, Foster J, 2nd, Cao L, et al. FAM65B is a membrane-associated protein of hair cell stereocilia required for hearing. Proc Natl Acad Sci U S A. 2014; 111: 9864-8.

42. Zhao B, Wu Z, Muller U. Murine Fam65b forms ring-like structures at the base of stereocilia critical for mechanosensory hair cell function. Elife. 2016; 5 .

43. Diaz-Horta O, Abad C, Cengiz FB, Bademci G, Blackwelder P, Walz K, et al. Ripor2 is involved in auditory hair cell stereociliary bundle structure and orientation. J Mol Med (Berl). 2018; 96: 1227-38.

44. de Bruijn SE, Smits JJ, Liu C, Lanting CP, Beynon AJ, Blankevoort J, et al. A RIPOR2 in-frame deletion is a frequent and highly penetrant cause of adult-onset hearing loss. J Med Genet. 2020.
45. Asensio-Juarez G, Llorente-Gonzalez C, Vicente-Manzanares M. Linking the Landscape of MYH9-Related Diseases to the Molecular Mechanisms that Control Non-Muscle Myosin II-A Function in Cells. Cells. 2020; 9.

46. Janke C, Montagnac G. Causes and Consequences of Microtubule Acetylation. Curr Biol. 2017; 27: R1287-R92.

47. Chandrashekar DS, Bashel B, Balasubramanya SAH, Creighton CJ, Ponce-Rodriguez I, Chakravarthi B, et al. UALCAN: A Portal for Facilitating Tumor Subgroup Gene Expression and Survival Analyses. Neoplasia. 2017; 19: 649-58.

48. Zhang K, Waxman DJ. PC3 prostate tumor-initiating cells with molecular profile FAM65Bhigh/MFI2low/LEF1low increase tumor angiogenesis. Mol Cancer. 2010; 9: 319.

49. Wu C, Orozco C, Boyer J, Leglise M, Goodale J, Batalov S, et al. BioGPS: an extensible and customizable portal for querying and organizing gene annotation resources. Genome Biol. 2009; 10: R130.

50. Jansen S, Gosens R, Wieland T, Schmidt M. Paving the Rho in cancer metastasis: Rho GTPases and beyond. Pharmacol Ther. 2018; 183: 1-21. 